\title{
La retroalimentación del modelo de agronegocios. Dieta, poder y cambio climático en el agro pampeano (1960-2008) ${ }^{1}$
}

\author{
Luis Ernesto Blacha ${ }^{2}$
}

\begin{abstract}
Resumen
Los usos del territorio que promueve el modelo de agronegocios inciden en la calidad de los alimentos obtenidos y en la sustentabilidad del ambiente. A partir de un enfoque sociológico contemporáneo, este trabajo se propone caracterizar y analizar una racionalidad donde el monocultivo degrada la dieta y simplifica los ecosistemas. Se trata de un modelo productivo que distancia a productores de consumidores, incrementando tanto la energía necesaria para producir una caloría de alimento como aquella que se consume en procesar las materias primas para que puedan extender su vida útil. Las transformaciones técnicas de la Revolución Verde -a comienzos de la década de 1960- son el punto de partida de un proceso que incrementa su productividad a expensas de la biodiversidad. Se consolida así la industrialización de la agricultura fundamentada en el carácter arquitectónico del poder, que resulta en alimentos con menor calidad nutricional que tienden a la malnutrición de la población. Los agronegocios son parte de este proceso, donde los problemas generados se convierten en insumos para seguir funcionando y permiten, a su vez, explicar el nexo dieta, poder y cambio climático.
\end{abstract}

Palabras clave: dieta - poder - territorio alimentos - agronegocios

\begin{abstract}
The uses of the territory promoted by the agribusiness model affect the quality of the food obtained and the sustainability of the environment. Based on a contemporary sociological approach, this work proposes to characterize and analyze a rationality where monoculture degrades the diet and simplifies ecosystems. It is a productive model that distances producers from consumers, increasing both the energy needed to produce a calorie of food and that consumed in processing raw materials so that they can extend their useful life. The technical transformations of the Green Revolution -at the beginning of the 1960s- are the starting point of a process that increases productivity at the expense of biodiversity. This consolidates the industrialization of agriculture based on the architectural character of power, resulting in foods with lower nutritional quality that tend to malnutrition of the population. Agribusiness is part of this process, where the problems generated become inputs to continue functioning and allow, in turn, to explain the nexus diet, power and climate change.
\end{abstract}

Keywords: diet - power - territory - food agrobusiness

${ }^{1}$ Trabajo recibido el 22/10/2018. Aceptado el 03/12/2018.

${ }^{2}$ Universidad Nacional de Quilmes. Consejo Nacional de Investigaciones Científicas y Técnicas. Contacto: luisblacha@gmail.com 


\section{Introducción}

Los adelantos técnicos que se englobaron en la Revolución Verde permitieron un incremento cuantitativo de la producción agrícola, primero y pecuaria luego, que transformaron el mundo rural. La mecanización de la producción junto al uso de fertilizantes y pesticidas implicaron importantes cambios socioeconómicos, a partir de una mayor injerencia del capital financiero en las producciones agropecuarias, que resultaron en nuevas formas de organización. Las herramientas de esta Revolución se complejizaron y extendieron en el tiempo, resultando en el punto de partida para el desarrollo e implementación del modelo de agronegocios que resignificó el vínculo entre productores y consumidores de alimentos.

La propuesta de este trabajo es caracterizar y analizar la relación entre las transformaciones en la dieta de la población argentina de finales del siglo XX y principios del siglo XXI, con los nuevos usos del territorio que auspician los agronegocios y que van más allá de la «agriculturización» de la región pampeana, eje histórico del modelo agroexportador imperante. El recorrido se inicia en la década de 1960 con la Revolución Verde porque es cuando se activaron las herramientas técnicas y los avances tecnológicos que permitieron una intensiva simplificación de los ecosistemas para incrementar, en el corto plazo, la productividad a costa de la biodiversidad. Se trató de un salto cuantitativo en la producción agrícola que no encontró su correlato en la calidad nutricional de los alimentos producidos. Hay una degradación nutricional de la comida (Winson, 2013) que para el caso argentino lleva a la pérdida del patrón alimentario unificado en la década de 1990 (Aguirre, 2004). Un patrón alimentario unificado hace referencia a que «ricos y pobres pensaban la comida de forma similar» porque ambos grupos tenían acceso a los alimentos para satisfacer sus necesidades nutricionales. (Aguirre, 2004, p. 30)

Los nuevos usos del territorio promovidos por el modelo de agronegocios coinciden con el pasaje de la desnutrición a la malnutrición como paradigma de las cadenas agroalimentarias. El monocultivo es su forma característica y pretende incrementar la productividad a partir de una reducción de la biodiversidad que termina debilitando el contexto ambiental. Este artículo propone caracterizar y analizar, en perspectiva sociológica, algunas de las transformaciones menos estudiadas del modelo de agronegocios: el vínculo entre la degradación de la dieta (malnutri- 
ción) y el cambio climático a partir de los usos del territorio, tomando como referencia el agro pampeano.

Las dietas «reflejan las condiciones materiales bajo las cuales los seres humanos han existido en un período determinado de tiempo» (Winson, 2013, p. 26). En el modelo de agronegocios, los alimentos frescos pasan a ser industrializados y bien conservados a partir de procesos mecánicos que los convierten no en «'buenos para comer' sino en 'buenos para vender'» (Aguirre, 2004, p. 10). Es el poder, como apropiación social del espacio a partir de saberes pero también porque la vida se convierte en objeto de gobierno, la herramienta que permite explicar el desarrollo de conocimientos e instrumentos técnicos que transforman tanto el ambiente como la dieta y el cuerpo humano. La interdependencia de esos factores lleva a los agronegocios a realimentarse de los problemas ambientales -aunque apele al lenguaje de la sociedad del conocimiento- $\mathrm{y}$ de las desigualdades sociales que su propio funcionamiento genera.

\section{De la agricultura a los agronegocios: los usos del espacio}

A mediados del siglo XX se expandió por Latinoamérica un modelo productivo, nacido en Estados Unidos durante la Segunda Guerra Mundial, que transformó al mundo rural mediante un aumento de la producción de alimentos a partir de la mecanización y del uso intensivo de fertilizantes y pesticidas. El saber, como insumo del poder, modificó los elementos que conformaban el ambiente e indujeron a inundaciones y/o sequías como sus principales características, aunque no las únicas. La interacción de animales, plantas y seres humanos se vio interpelada por un tipo de saber que promovía una mayor simplificación de los ecosistemas para incrementar la productividad. Estos cambios fueron económicos, ambientales, políticos pero también sociales y se los conoce como Revolución Verde. Hubo un incremento de la productividad a expensas de la reducción en la biodiversidad, que redefinió la manera de interpretar el mundo y el fundamento del orden social. Surgieron nuevos usos del territorio, vinculados al monocultivo, que ya no dependían exclusivamente de la propiedad de la tierra sino del saber y la tecnología implicados en potenciar su productividad.

La apropiación social del espacio se fundamenta en los vínculos de poder y la gubernamentalidad (Foucault, 2007). A partir de un conjunto 
de saberes, el poder diferencia cuáles son las interacciones que se desarrollan en un ámbito determinado de aquellos elementos que deben ser erradicados. Tal como sucede con el biopoder, dicha apropiación se convierte tanto en una necesidad como en una consecuencia del sistema productivo capitalista, que refleja el grado de complejización del entramado social que lo contiene. Así se define el territorio en tanto se convierte en condición indispensable para la consolidación del sistema productivo.

El vínculo saber-poder (Deleuze, 2014) fundamenta el carácter arquitectónico de los vínculos sociales del poder. Tal como teoriza Michel Foucault (2012), se multiplican aquellas cuestiones que interesan al poder. Surge una racionalidad propia de la Modernidad, que para finales del siglo XX se radicalizó como consecuencia de la complejización del entramado social y del alto grado de especialización de sus actores. La Modernidad amplió las fronteras temporales y espaciales de las interacciones sociales, en tanto la Modernidad Tardía de finales del siglo XX multiplicó aún más el contexto de interacción, hasta convertirlo en global (Giddens, 1997). Se consolidó una nueva geografía determinada por circuitos productivos y no por cercanías o vínculos precedentes (Santos, 2000).

Esta racionalidad ya estaba presente en la primera globalización de las cadenas agroalimentarias de finales del siglo XIX; aquella que convirtió a América Latina en productora de bienes primarios para las principales metrópolis europeas. Fue una apropiación social del espacio con fines productivos, tal como refleja el vínculo Londres-Buenos Aires que da forma de embudo al trazado de las vías férreas y rutas argentinas que privilegiaban la inserción en el mercado mundial sobre la consolidación de uno interno, por cierto restringido, para una Argentina de casi 3 millones de kilómetros cuadrados. La Revolución Verde fue una nueva etapa en este proceso, el inicio de una segunda globalización de las cadenas agroalimentarias, a partir de un incremento en la capacidad del hombre para modificar su ambiente utilizando ciertos saberes específicos e incorporando los combustibles fósiles como principal fuente de energía.

La fertilidad diferencial de la región pampeana -núcleo del modelo agroexportador- permitió alternar ciclos ganaderos y agrícolas, de acuerdo con la variación de los precios internacionales y el sistema de tenencia de la tierra (Barsky y Gelman, 2012). Con la supremacía de las demandas del mercado mundial, desde la primera globalización de las cadenas agroalimentarias, la producción local se desancló de sus continuidades espaciales, resultando influenciadas por decisiones tomadas del 
otro lado del océano Atlántico. El espacio termina por resignificarse, en lo que Anthony Giddens define como desanclaje/reanclaje (Giddens, 1997). Se reanclan las consecuencias de esta inserción en el mercado mundial como productores de materias primas agropecuarias. Es una vinculación donde «la localidad se opone a la globalidad, pero también se confunde con ella» (Santos, 2000, p. 274).

Los agronegocios son entendidos como «una serie de operaciones que se inician en la investigación y desarrollo, atraviesan el agro, la industria, el comercio y demás servicios anexos para atender las demandas de los consumidores» (Vilella y Senesi, 2009, pp. 7-8). A medida que el proceso se complejizó también lo hicieron sus consecuencias, tal como con la creciente pérdida de energía que lleva la industrialización de la agricultura, donde para producir una caloría de alimento se consumen 10 en su procesamiento, distribución y preparación (Bello, 2009, p. 148). Este funcionamiento del modelo de agronegocios ha llevado a un constante incremento de la productividad para poder continuar funcionando a pesar de consumir más energía de la que genera. Se trata de un modelo que prioriza la productividad, buscando un crecimiento continuo que resulta imposible en un mundo que es atrópico (Aledo y Sulaiman, 2014).

A diferencia de los ecosistemas naturales/originales, los agroecosistemas son conjuntos simplificados en donde se eligen qué elementos producir mientras que otros pasan a ser malezas o plagas (Reboratti, 2000). Es parte del carácter arquitectónico del poder, que encontró su correlato en el entramado social y en el rol ocupado por la Argentina en el mercado mundial. Tal como destacaba durante los años '70 la Teoría de la Dependencia, a finales del siglo XIX se conformó una nueva división mundial del trabajo en la cual los países del centro industrializaban las materias primas producidas por los países de la periferia (Sunkel, 1981). Una racionalidad que también se reprodujo al interior de cada Estado-Nación. Los ecosistemas en ámbitos periféricos se simplificaron para satisfacer las necesidades de los países centrales. La única manera de insertarse en el mercado mundial fue a costa de la biodiversidad. La Revolución Verde de mediados del siglo XX puso disposición de los productores un conjunto de saberes, como las nuevas semillas híbridas, fertilizantes y pesticidas, que redujeron aún más la biodiversidad para incrementar el rendimiento por hectárea. Con estas prácticas como punto de partida, los agronegocios proponen un modelo donde las necesidades y el gusto de los consumidores determinan cómo y qué se cultiva. Es el paradigma del monocultivo, como se observa en la región pampeana con 
la producción de soja genéticamente modificada desde 1996 y que en las décadas siguientes avanza hacia el Nordeste de la Argentina.

En el pasaje de la Revolución Verde al modelo de agronegocios hay un cambio en la escala de intervención del hombre en el ambiente. Los nuevos ecosistemas se caracterizan por su excesiva simplificación, lo que que los convierte en más propensos a sufrir epidemias (Leff, 2011). Estas transformaciones son posibles porque el poder politiza las cuestiones biológicas fundamentales, tanto en la vida humana como animal y vegetal. Cualidades y cantidades que pueden ser abordadas como parte de la gubernamentalidad que es la economía propia del arte de gobernar (Foucault, 2012). Lo paradógico de los agronegocios es que no contempla ni prácticas alternativas ni un diálogo con aquellos actores que forman parte de la «resistencia» y que Foucault identificaba como componente esencial de todos los vínculos de poder (Foucault, 1999). No pareciera haber propuestas alternativas, aún en casos inexplicables como el del etanol, cuya producción consume más petróleo que el combustible que genera (Giraldi, 2011).

La apropiación social del espacio a partir del vínculo saber-poder consolida a un sector agroalimentario que representa la convergencia de las producciones primarias con ciertas agroindustrias. Un sistema agroalimentario que puede caracterizarse como el «conjunto de actividades que desarrolla una sociedad para resolver sus necesidades básicas de alimentación» (CEPAL, FAO, IICA, 2017, p. 219). Es un entramado de interacciones sociales con implicancias económicas, culturales y productivas que se desarrolla a lo largo del tiempo y que hace suyo los nuevos usos del territorio, como sucede con el monocultivo. En el caso pampeano hay un importante avance de la agricultura que implicó, a comienzos del siglo XXI, el desplazamiento de 5 millones de hectáreas que eran ocupadas por la ganadería (Barsky y Gelman, 2009).

La evolución del sector agroalimentario puede abordarse como parte de un proceso, donde se combinan continuidades y cambios tanto en las formas de producir como de aquello que se produce. Se conjugan prácticas y actores que remiten a distintos contextos y necesidades, permitiendo identificar temporalidades superpuestas porque no todos los actores pueden ni desean incorporar con la celeridad los avances del sector agroalimentario. Esta distinción es notoria cuando se comparan las realidades de aquellos procesos cuyo destino es el mercado internacional mientras quedan relegadas las economías regionales y el mercado inter- 
no. Es parte del desanclaje que propone una geografía basada en circuitos productivos y que permite hablar de un proceso agroalimentario.

Es a partir del saber que se regulan los espacios, se delimitan las interacciones y se definen los actores. El poder transforma estos conocimientos en prácticas, como sucede en el mundo rural con la biotecnología que «permite superar muchos de los impedimentos bióticos y abióticos que hoy impiden aumentar la productividad de los cultivos.» (Trigo y Villarreal, 2010, pp. 162-163). Los vínculos entre el conocimiento y el territorio deben llevar al ajuste entre «nuestra tecnosfera a los límites que fija la biosfera, puesto que los procesos lineales de la primera, aunque innovadores, son ecológicamente inarmónicos o desajustados con la segunda, cuyos procesos son cíclicos, conservadores y autocoherentes» (Lecaros Urzúa, 2013, p. 181).

Hay una tensión entre desarrollo y sustentabilidad que es característica de la industrialización de la agricultura, porque la simplificación de los ecosistemas y la degradación de la dieta son problemas que los agronegocios convierten en insumos para seguir creciendo, como parte de su estrategia de acción. El capital financiero adquiere gran importancia en este modelo, tanto en las producciones primarias como en la distribución global de los productos agropecuarios, al punto de que «ni siquiera países con un potencial agrícola extraordinario tienen garantías de poder desarrollar un importante sector agroindustrial» (Barciela, 2017, p. 34). El incremento de la producción que propuso la Revolución Verde solo podía materializarse si era invertida una gran cantidad de dinero para incorporar los nuevos adelantos tecnológicos e industrializar la agricultura. Se trató de un proceso donde la reducción en la biodiversidad y la mayor estandarización de los procesos implementados también se tradujo en la homogeneización de los alimentos obtenidos. El capital financiero -que se instaló con firmeza durante el avance neoliberal de los años 90- pareciera promover una misma receta para todo tiempo y lugar, cuando los contextos socioeconómicos son diversos y están compuestos por actores que remiten a distintas coyunturas del mundo rural. Esta diversidad de actores y formas de producir permiten caracterizar el entramado social donde se insertan los agronegocios como temporalidades superpuestas, ya que es un modelo que intenta transformar prácticas precedentes sin tener en cuenta los contextos que los originaron.

Estos cambios fueron posibles porque se consolidó «un nuevo modelo de agricultura crecientemente intensivo en capital que se tradujo en constante aumentos de la producción y la productividad» (Barciela, 
2017, p. 24). Dichas cuestiones cobraron mayor impacto cuando la concentración de capital, el uso intensivo de los recursos naturales y la incorporación de innovaciones tecnológicas que expulsan mano de obra y modifican los usos del espacio tuvieron lugar en el contexto neoliberal de finales del siglo XX. Los sistemas agroindustriales que adquirieron mayor énfasis con la Revolución Verde indicaron cambios en los ciclos del carbón y el nitrógeno que impactaron en el cambio climático (Cleveland, 2014).

El modelo de agronegocios remite a una lógica que se retroalimenta o realimenta ${ }^{3}$ de sus propios resultados, aún de aquellos problemas que genera, como la simplificación de los ecosistemas y la homogeneización de los alimentos producidos que degradan la dieta de las sociedades industriales (Winson, 2013). También supone una respuesta en un contexto global donde son necesarios conocimientos que resulten en prácticas que «ordenen» el espacio para producir alimentos para $7 \mathrm{mil}$ millones de seres humanos que viven mayoritariamente en ciudades. Estilos de vida diversos parecieran unificarse a partir de aquello que comemos y donde la injerencia del capital financiero que promueve la industrialización de la agricultura borra las diferencias mediante un vacío social y cultural de los vínculos con el espacio desde mediados de la década de 1990 cuando se globalizan las grandes cadenas de supermercados.

El biopoder foucaultiano, como la politización de los rasgos biológicos fundamentales del hombre, pierde capacidad explicativa si no se incorporan otras formas de vida que también integran el ambiente. Las transformaciones en los ecosistemas también impactan en los alimentos. En la «politización» que reconstruye Foucault (2007) también debe considerarse el impacto de la malnutrición que promueven los alimentos basados en riego intensivo, con fuerte uso de fertilizantes y pesticidas y que remiten a cadenas productivas largas, es decir, donde productores y consumidores pueden encontrarse en distintos continentes.

La Revolución Verde prometía, en la décadas de 1960 y 1970, erradicar la desnutrición a partir del incremento en la producción, pero terminó modificando la composición de los alimentos. Los vegetales adquirieron mayor porcentaje de agua, como resultado del riego intensi-

\footnotetext{
${ }^{3} \mathrm{Si}$ bien la RAE no incorpora el término «retroalimentación», este hace referencia al concepto inglés de feedback, tomado de la terminología de la electrónica, en donde la salida de un sistema o circuito retorna a su origen. Para mayores referencias sobre realimentación ver: http://dle.rae.es/srv/search?m=30\&w = realimentar
} 
vo, y las carnes de feedlot mayor cantidad de grasas saturadas y omega 6 derivadas de la alimentación del ganado basada en granos. Como hay mayor cantidad de materias primas disponibles, la industrialización de la agricultura puede «ultraprocesarlas» para convertirlas en alimentos que se parecen muy poco a sus versiones originales. Se vuelven más atractivos para el consumidor porque son más dulces, grasosos y salados, generando una mayor satisfacción pero degradando la dieta y los cuerpos de esos consumidores.

La malnutrición que resulta de estos procesos, y que el modelo de agronegocios expande de forma exponencial, no es una condición exclusiva de las sociedades industriales, ni de las avanzadas ni de aquellas en vías de desarrollo, sino una cuestión que afecta al planeta en su conjunto (Latour, 2017). Es una cuestión ambiental cuyo origen es eminentemente social. No solo por el carácter interdependiente de los ecosistemas, que «silencian primaveras», sino porque también los sistemas productivos dependen unos de otros (Carson, 2016).

El vínculo entre el monocultivo, la degradación de la dieta y el cambio climático remite no solo a cuestiones técnicas sino a transformaciones sociales que deben abordarse desde la sociología, tomando como punto de partida el poder que permite implementar estas prácticas transformadoras. De hecho, la sociedad se complejiza porque los sujetos se individualizan llevando a una coyuntura donde a mayor desarrollo de la sociedad hay un mayor desarrollo del individuo. En esa individuación también se moldea nuestro gusto y nuestras preferencias sobre aquello que comemos (o dejamos de comer) que van a determinar qué se culti$\mathrm{va} / \mathrm{crí}$. Resulta una paradoja que la complejización de la sociedad tenga su antítesis en la naturaleza: cuando más compleja es una sociedad más simplificados resultan los ecosistemas que la sostienen. Estas transformaciones implican una red interdependiente de vínculos sociales que desafían la concepción convencional de desarrollo y que van más allá de la noción clásica del dominio del hombre sobre la naturaleza (Leff, 2001).

La escisión del sujeto agrícola -con la aparición del contratista, dueño del capital y la tecnología- que se produjo en la región pampeana, a partir de la década de 1970, dio lugar a un proceso donde quienes poseían la tierra no necesariamente contaban con el capital y el conocimiento para hacerlo producir e incorporar los nuevos avances técnicos. A partir de esta racionalidad, en Latinoamérica, pero también en Asia y Estados Unidos, se iniciaron procesos tecnológicos sobre la base de tres pilares: «a) la biotecnología; b) la oferta creciente de agroquímicos; y c) 
los permanentes progresos derivados de la ingeniería genética» (García et al., 2008, p. 170). La producción demandó mayor cantidad de capital para poder incorporar insumos de avanzada, compitiendo por el acceso a divisas con la industrialización por sustitución de importaciones (ISI).

Prácticas «obsoletas» y actores «improductivos» conviven con los «exitosos» exponentes de los agronegocios, aún antes de la incorporación de cultivos genéticamente modificados (Gras y Hernández, 2016). Esta diversidad de actores hace posible entender el entramado de los agronegocios como parte de las temporalidades superpuestas de la Modernidad radicalizada de finales del siglo XX. El año 1996 fue paradigmático para el caso argentino porque se implementó la primera campaña oficial con soja RR (Round-up Ready) y se delineó - junto a la siembra directa- una racionalidad donde productores y consumidores se vinculaban a partir de las grandes empresas transnacionales de alimentos. El neoliberalismo de finales del siglo XX propuso un único modelo agroindustrial para los distintos entramados sociales del mundo.

Estas nuevas tecnologías, impulsadas por el modelo de agronegocios, permitieron que la región pampeana argentina extendiera su frontera agrícola y se triplicara el área cosechada con granos (de 10 a 30 millones de has.) pero también que su producción se multiplicara por ocho y el rendimiento promedio por hectárea creciera $150 \%$ desde mediados de la década de 1990 hasta el 2005 (Reca, Lema y Flood, 2010). Simultáneamente, la siembra directa permitió un ahorro de casi $60 \%$ en combustible y menor demanda de mano de obra. Estos cambios convirtieron al «antiguo granero del mundo en un productor de forraje para el ganado europeo [y chino] y con tasas de crecimiento superiores a la media del mundo» (Robin, 2016, p. 301).

De exportar alimentos por 1300 millones de dólares en 1970, Argentina pasa a exportar 35.000 millones en 2008 (Scheinkerman de Obschatko, 2010). Este importante incremento en la capacidad de producir alimentos no hace referencia a la calidad de los mismos ni a su composición nutricional. El pasaje de la agricultura a los agronegocios también debe incorporar las modificaciones en la dieta que lleva a cambios físicos en sus consumidores, como la obesidad, y a su degradación nutricional que resulta en la malnutrición. 


\section{Dieta y cambio climático}

La receta universal -pesticidas, mecanización, fertilizantes y semillas genéticamente modificadas y capital financiero- propuesta por el pasaje de la agricultura a los agronegocios fue posible por la consolidación de sistemas expertos que subrayaron los saltos productivos y dejaron en un segundo lugar la sustentabilidad. La productividad basada en la simplificación de los ecosistemas se incrementó con el uso de fertilizantes y pesticidas conjuntamente con semillas modificadas. Como aquellos están basados en derivados del petróleo aumenta la dependencia del mundo rural de esta fuente de energía no renovable. También crece la distancia geográfica entre productores y consumidores, que multiplican las food miles; es decir, que aumentan los kilómetros que viajan los componentes de un plato típico desde su producción hasta que es servido en la mesa de los comensales.

En la actualidad un plato de comida en Europa involucra que sus componentes viajen 2400 kilómetros (Clapp, 2016); en el caso argentino solo un litro de leche debe recorrer 1500 kilómetros (Portal lechero, 2018). Además, la soberanía, en tanto capacidad de decidir sobre un territorio, se encuentra interpelada por nuevos actores -las empresas transnacionales de alimentos- que también inciden en el régimen alimentario. Si para alimentar a más seres humanos se depende de menos especies animales y vegetales, aquellas que forman parte de la dieta deben tener gran productividad y cualquier reducción en los resultados esperados puede llevar al hambre (Rieff, 2016).

El costo de estas transformaciones es también ambiental, tal como refleja que en el mundo existan «2000 millones de hectáreas de paisajes deforestados y degradados que se deben restaurar» (CEPAL, FAO, IICA, 2017). Los alimentos se desanclan de su lugar de origen y van allí donde puedan ser vendidos al mayor precio, pero las consecuencias de su producción y distribución se reanclan en sus espacios primarios. Los sistemas expertos que permiten este uso del territorio no pueden evitar el riesgo y se basan en una calculabilidad limitada que termina potenciando el carácter transformador de las interacciones sociales. El proceso agroalimentario vincula la degradación de la dieta y del ambiente en un entramado donde «la primera ley de los riesgos medioambientales es: la contaminación sigue al pobre» (Beck, 2002, p. 8). Como los alimentos se convierten en un commodity, el hambre también pareciera estar muy cerca de aquellos que no cuentan con el dinero para comprarlos. Tal como 
ha sucedido con la suba de los alimentos a nivel mundial en 2007 (Rieff, 2016).

Además del monocultivo sojero, el modelo de agronegocios impulsa la cría de ganado bovino a corral o feedlot como reemplazo del pastoreo tradicional. Una práctica que consiste en la aglomeración de ganado en espacios reducidos que resulta en un importante daño ambiental, ya que este tipo de animales «elimina como estiércol un 5 a $6 \%$ de su peso vivo por día. Es decir que un engorde a corral que tenga un peso promedio por animal de 200 kilos tendría: 100 animales, 1 tn de estiércol/día...» (Adámoli, 2006, p. 1). El feedlot actúa como un mercado donde colocar el excedente de la producción de granos para alimentar a los animales, necesitando de $« 6 \mathrm{~kg}$ de maíz para producir un $\mathrm{kg}$ de carne bovina» (Gimeno, 2010, pp. 349-350). Son nuevos usos del territorio que la arquitectura del poder delinea para incrementar la productividad sobre la sustentabilidad.

La mayor producción en menos espacio y reduciendo costos se fundamenta en la lógica capitalista que necesita de una gubernamentalidad que sostenga un mercado consumidor donde estén disponibles los alimentos obtenidos. El biopoder se convierte en la herramienta analítica capaz de explicar esta racionalidad que vincula los usos del territorio con los cambios en la dieta y en el ambiente. Como el biopoder se fundamenta en el saber, son los conocimientos técnicos los que permiten el monocultivo pero también la estructura administrativa y la distribución de alimentos en amplios espacios geográficos. A diferencia de la propuesta foucaultiana, las cadenas agroalimentarias combinan los esfuerzos normativos del Estado para defender la salud de la población con la homogeneización que resulta del modelo de agronegocios.

La gubernamentalidad como aplicación del biopoder recurre a la estadística como una herramienta específica para abordar las distintas variables que componen a la población. A partir del conocimiento estadístico puede reconstruirse una mirada antropométrica que tenga en cuenta el efecto de, por ejemplo, los agronegocios en las tallas de un conjunto de individuos. La antropometría lleva a descubrir que hay un vínculo entre las tallas de la población y la bonanza o las crisis de una sociedad, porque el acceso a proteínas (principalmente de origen animal) permiten ganar centímetros, mientras que el trabajo extenuante y las enfermedades en la infancia restan centímetros a la estatura. Más allá de la carga genética, el descenso en la mortalidad infantil permite a una población ganar centímetros con rapidez, pero llegada una altura (cerca- 
na al 1,72 ) va a llevar más tiempo, porque se requieren activas políticas públicas que velen por la nutrición y la salud de esa población (GalofréVilà, Martínez Carrión y Puche, 2018). En contrapartida, la malnutrición resulta en la incapacidad de una persona por obtener «suficientes vitaminas, sales, minerales o proteínas, incluso si la cuenta calórica de lo consumido es más que suficiente» (Rieff, 2016, p.157).

Desde las innovaciones de la Revolución Verde se ha incrementado la capacidad para producir alimentos para un mayor número de individuos. Los agronegocios, por su parte, incorporaron canales de distribución más dinámicos, como las grandes cadenas de almacenes y supermercados que también forman parte de este modelo. Hay una estandarización tal de la producción que surge la malnutrición, que «abarca desde la desnutrición crónica hasta el sobrepeso y la obesidad. Afecta a las poblaciones a lo largo del ciclo de vida, desde su concepción y durante la infancia hasta la adolescencia, la edad adulta y la vejez» (FAO, FIDA, OMS, PMA y UNICEF, 2018, p. 15). Los cambios en los cuerpos de los consumidores reflejan la «degradación nutricional de la comida». (Winson, 2013, p. 8) y «por primera vez en la historia de nuestra especie, la mayoría de los adultos en ciertas sociedades, y sorprendentes proporciones de chicos y jóvenes, se han convertido en «obesos» y están excedidos de peso» (Winson, 2013, p. 14). Hay un tránsito de la desnutrición a la malnutrición que da cuenta de la incidencia de los intereses económicos en las necesidades de la población y cómo éstas pueden manipularse a partir de la publicidad.

El suministro y procesamiento excesivo de los alimentos también son parte del modelo de agronegocios. Cuando comienza a solucionarse la desnutrición, el modo que adquiere la producción de alimentos promueve la malnutrición. Un proceso al cual no es ajeno el poder y que merece este abordaje sociológico; porque los pequeños productores pierden capacidad de decisión y son las grandes empresas transnacionales las que determinan qué y cómo se produce. Hay tal asimetría que el informe OxfamIntermón de 2013 muestra «una realidad que contabiliza 7000 millones de consumidores de alimentos y 1500 millones de productores, tan solo menos de 500 empresas controlan el $70 \%$ de la oferta de alimentos» (Melgarejo Moreno y Abadía Sánchez, 2017, p. 18). Este desequilibrio en la capacidad de qué producir termina delimitando el acceso a cierto tipo de alimentos, más saludables pero con menores índices de ganancia. Así ocurre con los alimentos ultra procesados industrializa- 
dos, a los que «a menudo se les da mayor volumen con aire o agua» (OPS, 2015, pp. 5-6).

El distanciamiento de los productores de alimentos respecto de los potenciales consumidores revela que comer «une lo biológico y lo cultural de una manera tan indisoluble que difícilmente podamos separarlos y esto arranca de las características mismas de la especie humana como especie social» (Aguirre, 2004, p. 2). La consolidación de cierta forma del sistema agroalimentario no debe ocultar su carácter histórico ni su dinamismo; cuestiones que le ha permitido transformarse en el pasado. No hay una receta que sea certera para todos los casos y es clave el rol de los sistemas expertos para convertir al sistema agroalimentario en posibilidad de cambio y no en el mantenimiento de ciertos intereses económicos y políticos- que atentan contra la sustentabilidad en el mediano plazo.

\section{Reflexiones finales: los problemas como insumos}

El recorrido propuesto ha utilizado algunas herramientas de la sociología contemporánea para complejizar el modelo de agronegocios más allá de una cuestión técnica con implicancias socioeconómicas. La elección de la perspectiva foucaultiana del biopoder ha permitido enunciar los usos del territorio como parte del carácter arquitectónico del poder que impacta tanto en las formas de producir como en los resultados obtenidos. Los importantes saltos cuantitativos que se iniciaron con la Revolución Verde fueron en detrimento de la calidad nutricional de los alimentos, priorizando la estandarización y el rendimiento por hectárea. Se modificaron genéticamente las semillas pero también los cuerpos de los consumidores resultaron transformados a partir de aquellos alimentos que conforman su dieta.

Con esa Revolución se inició una nueva etapa en la industrialización de la agricultura a partir de un cambio de escala en las lógicas productivas que ya estaban presentes en el mundo rural desde, por lo menos, finales del siglo XIX. Estos sistemas expertos permitieron reconocer la diversidad en cosechas, malezas y plagas para incrementar la productividad a costa de la biodiversidad. Hubo un pasaje de la agricultura a los agronegocios que incrementó la distancia entre productores y consumidores e implicó un cambio cultural, al punto que en los sistemas modernos agroalimentarios, el procesamiento y la distribución determinan 
el vínculo con los alimentos. Es parte del desanclaje-reanclaje que el sociólogo inglés Anthony Giddens describió como propio de la Modernidad y que se radicalizó a finales del siglo XX.

Hay una tensión entre desarrollo y sustentabilidad donde la capacidad de retroalimentarse del sistema productivo convierte los problemas en insumos. ¿Es la productividad un buen indicador si no se tiene en cuenta la sustentabilidad? Se trata de una pregunta que la propia inercia de los agronegocios pareciera responder a partir del modo en que se retroalimenta de los problemas de su propio funcionamiento para constituirse como el único camino posible. Es parte de estos múltiples tiempos, donde confluyen el concierto global con las perspectivas nacionales y regionales, junto con la escala local más cercana y para todos pareciera existir un único camino posible: el modelo de agronegocios. Es un entramado donde las transformaciones más globales, como el cambio climático, conviven con la degradación de la dieta que se presenta como una cuestión meramente personal.

El carácter oligopólico de las grandes empresas de la alimentación también tuvo su punto de partida con la Revolución Verde y la mayor injerencia del capital financiero en el mundo rural. Todas estas cuestiones forman parte del poder acerca del cual teoriza Michel Foucault y que requiere de renovadas funciones, porque los actores y su modo de vincularse con el entorno se han transformado. Además de la salud, debe estar presente el acceso a los alimentos y cómo se producen para no dañar el ambiente ni deteriorar la calidad de vida de la población. Una cuestión que también interpela a la política, ya que si en la región pampeana las calorías producidas se distribuyeran equitativamente a cada habitante le corresponderían $3181 \mathrm{kilo/calorías} \mathrm{por} \mathrm{día;} \mathrm{es} \mathrm{decir,} \mathrm{casi} 20$ veces más de las necesarias para un hombre adulto. Sin embargo la escasa diversidad de alimentos y su reducido aporte nutricional llevan a cuestiones críticas como el síndrome metabólico en donde el cuerpo demanda -casi como una adicción- aquellos alimentos que lo llevan a enfermarse (Lusting, 2017). Se trata de una cuestión que cobra cada vez más visibilidad en la salud pública y se convierte en origen de la obesidad, la diabetes y la malnutrición.

El acceso y la distribución de alimentos es una cuestión central en el modelo de agronegocios que también debe ser tomada en cuenta; de allí la necesidad de su estudio. Es una oportunidad para ir más allá de la retroalimentación que lo mantiene en funcionamiento sin cuestionamientos. Casi la mitad de los residuos están conformados por alimentos, con- 
virtiéndose en una alternativa a la racionalidad imperante. Si bien cómo se produce termina determinando quién realiza esa producción y cómo son los resultados obtenidos, abordar el modelo de agronegocios en conjunto con las cadenas de supermercados y las transformaciones en la dieta permite complejizar el análisis. Un conjunto de alternativas entre las que deben incluirse la policultura, las cosechas biodinámicas y las certificaciones de producción y origen como un modo de generar valor agregado a la producción en base a la calidad de los alimentos. Desde la sociología no hay que olvidar que el principal escollo para que cambie el sistema agroalimentario es el propio sistema agroalimentario, tal como está configurado en la actualidad; con sus intereses, sus vínculos ya creados y la cantidad de excluidos del acceso a una adecuada cuota alimentaria que lleva a la malnutrición.

El análisis del modelo de agronegocios, considerando su aporte al cambio climático y a la degradación de la dieta, es un abordaje novedoso que representa una transferencia social desde el conocimiento sociológico a la caracterización de un proceso que se inicia con los usos del territorio rural pero que también va mucho más allá. Las cadenas globales de supermercados, como su lazo urbano, son parte de este proceso cuya eficiencia se fundamenta en vincular productores y consumidores, transformándolos a ambos. El pequeño productor familiar es desplazado por el monocultivo de soja y un conjunto de prácticas que delimitan cómo producir. Esto termina incidiendo en la composición de los alimentos obtenidos: altos en azúcares y grasas pero con bajo valor nutricional. Como resultado se modifican los cuerpos, a partir de un vacío sociocultural que intenta convertir la comida en «inocua». Las herramientas sociológicas permiten no solo describir una racionalidad productiva sino también su impacto social y como ésta se retroalimenta de sus consecuencias para seguir funcionando.

\section{Referencias bibliográficas}

Adámoli, J. (2006). Aspectos ecológicos de la ganadería: im-pacto de la siembra directa y el manejo. Congreso Ganadero de AAPRESID. Recuperado de http://www.produccionbovina.com/sustentabilidad/ 51-impacto_siembra_directa.htm

Aguirre, P. (2004). Ricos flacos y gordos pobres. La alimentación en crisis. Buenos Aires, Argentina: Capital Intelectual. 
Aledo, A. y Sulaiman, S. (2014). La incuestionabilidad del riesgo. Ambiente \& Sociedade, 17(4), 9-16.

Barciela López, C. (2017). Los cambios en la cadena agroalimentaria mundial en las últimas décadas y la posición de España. En R. Abadía Sánchez y J. Melgarejo Moreno, El sector agroalimentario: sostenibilidad, cooperación y expansión, pp. 23-49. Alicante, España: Ayuntamiento de Orihuela y Universidad de Alicante.

Barsky, O. y Gelman, J. (2012). Historia del agro argentino. Desde la conquista hasta comienzos del siglo XXI. Buenos Aires, Argentina: Sudamericana.

Beck, U. (2002). La sociedad del riesgo global. Madrid, España: Siglo XXI. Bello, W. (2009). The Food Wars. New York, Estados Unidos: Verso.

Blacha, L.E. (2018). La Revolución Verde y la degradación de la dieta. Un enfoque sociológico del proceso agroalimentario pampeano. Revista Diálogos en Mercosur, (5), 114-133.

Carson, R. (2016). Primavera silenciosa. Barcelona, España: Crítica-Editorial Planeta.

CEPAL, FAO, IICA (2017). Perspectivas de la agricultura y del desarrollo rural en las Américas: una mirada hacia América Latina y el Caribe 2017-2018. San José, Costa Rica: IICA.

Clapp, J. (2016). Food (Resources). Cambrigde, Estados Unidos: Polity Press.

Cleveland, D. (2014). Balancing on a Planet: The Future of Food and Agriculture. California, Estados Unidos: Studies in Food and Culture Book 46.

Deaton, A. (2015). El gran escape. Salud, riqueza y los origenes de la desigualdad. México, México: Fondo de Cultura Económica.

FAO, FIDA, OMS, PMA y UNICEF (2018). El Estado de la seguridad alimentariay la mutrición en el mundo 2017. Fomentando la resiliencia en aras de la paz y la seguridad alimentaria. Roma, Italia: FAO

Foucault, M. (1999). Historia de la sexualidad 1. La voluntad de saber. México, México: Siglo XXI.

Foucault, M. (2007). Seguridad, territorio y población: curso en el College de France: 1977-1978. Buenos Aires, Argentina: Siglo XXI.

Foucault, M. (2012). Nacimiento de la biopolítica. Curso en el Collège de 
France (1978-1979). Buenos Aires, Argentina: Fondo de Cultura Económica.

Galofré-Vilà, G., Martínez-Carrión, J.M. y Puche, J. (2018). Height and Climate in Mediterranean Spain, 1850-1949" en Journal of Interdisciplinary History, 49 (2), 247-277.

García, I.L, García, A.O, Rodríguez, E. y Rofman, A. (2008). Los dos «campos» en el territorio argentino: Análisis crítico y estrategias de desarrollo rural. Revista de estudios regionales y mercado de trabajo, (4), 167-200.

Giberti, H. (1981). Historia económica de la ganadería argentina. Buenos Aires, Argentina: Hyspamérica

Giddens, A. (1997). Consecuencias de la Modernidad. Madrid, España: Alianza Universidad

Giddens, A. (2000). La transformación de la intimidad. Sexualidad, amor $y$ erotismo en las sociedades modernas. Madrid, España: Cátedra.

Giddens, A. (2010). La política del cambio climático. Madrid, España: Alianza.

Gilardi, R. (2011). Cambio climático y soberanía alimentaria. En $M$. de Gorban et.al., Seguridad y soberania alimentaria, pp. 169-180. Buenos Aires, Argentina: Colección Cuadernos.

Gimeno, E. J. (2010). Aspectos de la sanidad bovina. En L. Reca, D. Lema y C. Flood (Eds.). El crecimiento de la agricultura argentina. Medio siglo de logros y desafíos. Buenos Aires, Argentina: Universidad de Buenos Aires.

Gras, C. y Hernández, V. (2016). Radiografía del nuevo campo argentino. Del terrateniente al empresario transnacional. Buenos Aires, Argentina: Siglo XXI.

Klein, N. (2015). Esto lo cambia todo. El capitalismo contra el clima. Buenos Aires, Argentina: Paidós.

Latour, B. (2017). Cara a cara con el planeta. Una nueva mirada sobre el cambio climático alejada de las posiciones apocalipticas. Buenos Aires, Argentina: Siglo XXI

Lecaros Urzúa, J.A. (2013). La ética medio ambiental: principios y valores para una ciudadanía responsable en la sociedad global. Acta Bioethica, 19 (2), 177-188

Leff, E. (2001). Ecología y capital. Racionalidad ambiental, democracia participativa y desarrollo sustentable. México, México: Siglo XXI. 
Lusting, R. H. (2017). The hacking of the American Mind. The science behind the corporate takeover of our bodies and brains. New York, Estados Unidos: Avery

Megarejo Moreno, J. y Abadía Sanchez, R. (2017). Una introducción al sector agroalimentario. En R. Abadía Sánchez y J. Melgarejo Moreno, El sector agroalimentario: sostenibilidad, cooperación y expansión, pp. 15-21. Alicante, España: Ayuntamiento de Orihuela y Universidad de Alicante.

Organización Panamericana de la Salud. Alimentos y bebidas ultraprocesados en América Latina: tendencias, efecto sobre la obesidad e implicaciones para las politicas públicas. Washington, Estados Unidos: Organización Panamericana de la Salud.

Ordóñez, H. (2009) La nueva economía y negocios agroalimentarios. Buenos Aires, Argentina: Facultad de Agronomía.

Portal Lechero. (2018). Entrevista a Eduardo Zurro «Argentina: un litro de leche en Argentina viaja $1.500 \mathrm{~km}$. Recuperado de https:/ /www.portalechero.com/innovaportal/v/12851/1/innova.front/argentina:-\%C2\%A8un-litro-de-leche-en-argentina-viaja-1500kms\%C2\%A8.html

Reboratti, C. (2000). Ambiente y sociedad. Conceptos y relaciones. Buenos Aires, Argentina: Ariel.

Reca, L., Lema, D. y Flood, C. (Eds.). (2010). El crecimiento de la agricultura argentina. Medio siglo de logros y desafíos. Buenos Aires, Argentina: Universidad de Buenos Aires.

Rieff, D. (2016). El oprobio del hambre. Alimentos, justicia y dinero en el siglo XXI. Barcelona, España: Taurus

Robin, M. (2016). El mundo según Monsanto. De la dioxina a los OGM: una multinacional que le desea lo mejor. Barcelona, España: Península.

Santos, M. (2000). La naturaleza del espacio. Técnica y tiempo. Razón y emoción. Barcelona, España: Ariel.

Scheinkerman de Obschatko, E. (2010). Desarrollo, estructura y posibilidades de la industria de alimentos y bebidas. En L. Reca, D. Lema, y C. Flood (Eds.), El crecimiento de la agricultura argentina. Medio siglo de logros y desafios. Buenos Aires, Argentina: Universidad de Buenos Aires 
Sunkel, O. (1981). La dimensión ambiental en los Estilos de Desarrollo de América Latina. Santiago de Chile, Chile: CEPAL.

Trigo, E. y Villarreal, F. (2010). La innovación biotecnológica en el sector agrícola. En L. Reca, D. Lema, y C. Flood (Eds.). El crecimiento de la agricultura argentina. Medio siglo de logros y desafios. Buenos Aires, Argentina: Universidad de Buenos Aires.

Vilella, F. y Senesi, S. (2009). Prólogo. En Ordóñez, H. (2009) La mueva economia y negocios agroalimentarios. Buenos Aires, Argentina: Facultad de Agronomía.

Walden, B. (2009). The food wars. New York-Londres, Estados Unidos, Inglaterra: Verso.

Winson, A. (2013). Industrial Diet: The Degradation of Food and the Struggle for Healthy Eating. Vancouver, Canadá: UBC Press.

Zapata, M.E., Rovirosa, A. y Carmuega, E. (2016). Cambios en el patrón de consumo de alimentos y bebidas en Argentina, 1996-2013. Salud colectiva, 12 (4), 473-486. doi: 10.18294/sc.2016.936 